\title{
Correction to: Supersingular K3 surfaces are unirational
}

\section{Christian Liedtke ${ }^{1}$}

Correction to: Invent. math. (2015) 200:979-1014 https://doi.org/10.1007/s00222-014-0547-7

As was pointed out by Bragg and Lieblich in [1], the proof of [2, Proposition 3.5] contains a mistake, which has the effect that the main result, the unirationality of supersingular K3 surfaces [2, Theorem 5.3], remains a conjecture.

\section{Degree p multisections}

More precisely, the proof only shows the following:

Proposition 0.1 We keep the notations and assumptions of [2, Proposition $3.2]$ and assume that $\widehat{\operatorname{Br}}(X)$ is not $p$-divisible. Set $R:=k[[t]]$ and $S:=\operatorname{Spec} R$, and let

$$
\bar{A} \rightarrow Y \times_{k} S \rightarrow S
$$

The original article can be found online at https://doi.org/10.1007/s00222-014-0547-7.

Christian Liedtke

liedtke@ma.tum.de

1 TU München, Zentrum Mathematik, M11, Boltzmannstr. 3, 85748 Garching bei München, Germany 
be a family of A-torsors associated to a non-trivial p-torsion element of $\widehat{\operatorname{Br}}(X)(R)$ as in [2, Proposition 3.2]. Then, after possibly replacing $S$ by some finite flat cover

(1) There exists a degree $p$ multisection $D_{\xi} \subset \bar{A}_{\xi}$ such that the induced morphism

$$
D_{\xi} \rightarrow \xi \times{ }_{k} S
$$

is finite and flat of degree $p$.

(2) More precisely, $D_{\xi} \rightarrow \xi \times_{k} S$ arises from a family of $A_{\xi}[p]$-torsors.

In particular, contrary to what was claimed in [2, Proposition 3.5], one may not be able to choose the degree $p$ multisection $D_{\xi}$ to be radicial, that is, purely inseparable, over $\xi \times{ }_{k} S$.

The strategy of establishing [2, Theorem 5.3] consisted in showing that any two supersingular K3 surfaces in characteristic $p \geq 5$ can be connected by one-dimensional families of the type as constructed in [2, Section 3] and then, that unirationality is preserved in these families. This reduced the unirationality conjecture to the showing that there exists at least one unirational K3 surface in each characteristic, where one can use Kummer surfaces, whose unirationality was established by Shioda [3].

Now, in order to show that unirationality is preserved in these onedimensional families, one needs Assertion (4) of [2, Theorem 3.6], whose proof requires that one can find degree $p$ multisections in [2, Proposition 3.5] that are radicial over the base. Without this, one can only conclude that any two supersingular K3 surfaces in characteristic $p \geq 5$ are connected by a sequence of very special degree $p$ correspondences (as in Assertion (3) of [2, Theorem 3.6]), which is not sufficient for unirationality.

\section{Fibration structure}

Moreover, the proof of [2, Theorem 4.3] contains a mistake. In fact, the proof only shows the following:

Theorem 0.2 Let $N$ and $N_{+}$be the supersingular K3 lattices in odd characteristic $p$ of Artin-invariants $\sigma_{0}$ and $\sigma_{0}+1$, respectively. Then, there exists a rank 20 lattice $\Lambda$, and isometries

$$
N \cong U \oplus \Lambda \text { and } N_{+} \cong U(p) \oplus \Lambda,
$$

where $U$ denotes the hyperbolic plane. This decomposition gives rise to a dominant rational map $\varpi_{N}$ of moduli spaces of rigidified $K 3$ crystals with a section $\sigma_{N}$ 


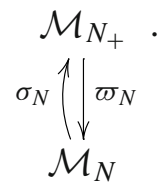

More precisely,

(1) this map is defined on the open set of $\mathcal{M}_{N+1}$ of rigidified K3 crystals with Artin invariant $\sigma_{0}+1$ and maps surjectively onto the open set of rigidified $K 3$ crystals of Artin invariant $\sigma_{0}$.

(2) The normalization of the geometric generic fibre is $\mathbb{P}^{1}$. (In general, $\varpi_{N}$ may not be generically smooth.)

In the proof, the map is only defined on geometric points and extension to a morphism would require more arguments. In particular, the computations in Step 6 of the proof only show that the normalisation of the geometric generic fibre is isomorphic to $\mathbb{P}^{1}$.

In particular, the table [2, Remark 4.4] has to be corrected as follows: for $\sigma_{0}(N)=3$, the moduli space $\mathcal{M}_{N}$ consists geometrically of two copies of the Fermat hypersurface of degree $(p+1)$ in $\mathbb{P}^{3}$. In this case, $\varpi_{N}$ arises geometrically by choosing a pencil of planes through a line of this surface and then, one obtains a fibration over $\mathbb{P}^{1}$, whose geometric generic fibre is a singular rational curve that is not smooth. This gives an example, where the geometric generic fibre of $\varpi_{N}$ is not smooth, and in particular, not isomorphic to $\mathbb{P}^{1}$.

\section{References}

1. Bragg, D., Lieblich, M.: Perfect points on genus one curves and consequences for supersingular K3 surfaces. arXiv:1904.04803 (2019)

2. Liedtke, C.: Supersingular K3 surfaces are unirational. Invent. math. 200, 979-1014 (2015)

3. Shioda, T.: Some results on unirationality of algebraic surfaces. Math. Ann. 230, 153-168 (1977)

Publisher's Note Springer Nature remains neutral with regard to jurisdictional claims in published maps and institutional affiliations. 